\title{
Article
}

\section{Impaired Adipose Tissue Expansion Caused by LXR Activation is Associated with Insulin Resistance in HFD Mice}

\author{
Yueting Dong ${ }^{1}$, Zhiye Xu ${ }^{1}$, Ziyi Zhang ${ }^{1}$, Xueyao Yin ${ }^{1}$, Xihua Lin ${ }^{2}$, Qianqian Pan ${ }^{1}$, Hong Li $^{1}$ and \\ Fenping Zheng ${ }^{1, *}$ \\ 1 Department of Endocrinology, Sir Run Run Shaw Hospital Affiliated with School of Zhejiang University, \\ 3 East Qingchun Road, Hangzhou 310016, China; dongyueting03@foxmail.com (Y.D.); \\ xuzhiye777@163.com (Z.X.); 3090101142@zju.edu.cn (Z.Z.); sharon_yin128@sina.com (X.Y.); \\ zhengfenping@tom.com (H.L.) \\ 2 Biomedical Research Center and Key Laboratory of Biotherapy of Zhejiang Province, \\ Sir Run Run Shaw Hospital Affiliated with School of Zhejiang University, 3 East Qingchun Road, \\ Hangzhou 310016, China; haxi1986@163.com \\ * Correspondence: zhengfenpingjindi@hotmail.com; Tel./Fax: +86-571-8600-2124
}

\begin{abstract}
Liver X receptors (LXR) are deemed as potential drug targets for atherosclerosis, whereas a role in adipose tissue expansion and its relation to insulin sensitivity remains unclear. To assess the metabolic effects of LXR activation, C57BL/6 mice on a high-fat diet (HFD) were treated with the dual LXR $\alpha / \beta$ agonist T0901317 (30 mg/kg per day) for 3 weeks. Differentiated 3T3-L1 was used for analysing the effect of T0901317 on glucose uptake.T0901317 reduced fat mass, accompanied by a massive fatty liver and lower adipokine levels in circulation of HFD mice. Increased adipocyte apoptosis and macrophage infiltration were found in epididymal fat of T0901317-treated HFD mice. In addition, T0901317 treatment promoted basal lipolysis, but blunted the anti-lipolytic action of insulin. Furthermore, LXR activation antagonized PPAR $\gamma$ target genes in epididymal fat and PPAR $\gamma$-PPRE binding activity in 3T3-L1 adipocytes. Although the glucose tolerance was comparable to that in vehicle-treated HFD mice, the insulin tolerance was significantly decreased in T0901317-treated HFD mice, indicating decreased insulin sensitivity by T0901317 administration, and which was further supported by impaired insulin signalling found in epididymal fat and decreased insulin-induced glucose uptake in 3T3-L1 by T0901317administration. These findings reveal that LXR activation impairs adipose expansion which contributes to decreased insulin sensitivity.
\end{abstract}

Keywords: Liver X receptor (LXR); Peroxisome proliferator-activated receptor (PPAR $\gamma$ ); Adipose expansion; Insulin resistance 


\section{Introduction}

Adipose tissue is a key regulator of energy balance, playing an active role in lipid storage and buffering, and synthesizing and secreting a wide range of fatty acids and adipokines into the circulation that influence systemic metabolism [1]. There is strong evidence showing that dysfunction of adipose tissue plays a critical role in the development of insulin resistance and diabetes mellitus [2-3]. A classical paradigm is that the more adipose tissue, the higher the prevalence of metabolic diseases, and it is this relationship that has interested researchers [4]. However, recent studies have suggested that the factor linking obesity and insulin resistance may not be the absolute amount of fat accumulated but the mismatch between energy surplus and storage capacity [5]. Allowing adipose tissue to store more lipids may prevent secondary metabolic complications caused by lipids being deposited in non-adipose organs. Thus, the ability of adipose tissue to expand and match the storage needs of energy surplus may be a key determinant in protection against the metabolic syndrome associated with obesity [6].

Adipose tissue mass is determined by processes governing adipocyte size and number [7]. The size of adipocytes increases because of increased storage of triglycerides from dietary sources or endogenous lipogenic pathways, whereas adipocyte number increases as a result of increased proliferation and differentiation [8]. Decreases in adipose tissue mass may involve the loss of lipids through lipolysis and the loss of mature fat cells through apoptosis [9]. Peroxisome proliferator-activated receptor (PPAR $\gamma$ ) is a critical regulator of adipose tissue mass. The activation of PPAR $\gamma$ leads to adipocyte differentiation and fatty acid storage [10-11]. Moreover, the expansion of adipose tissue associated with obesity may be based on a hyperplasic response of the adipose tissue regulated by PPAR $\gamma$ rather than just on hypertrophy of the mature adipocytes, thus resulting in adipose tissue with smaller but more numerous adipocytes. These smaller adipocytes retain insulin sensitivity with the secretion of insulin-sensitizing adipokines. This occurs in a mouse model that is heterozygous for PPAR $\gamma$, which shows improved insulin sensitivity and protection from lipotoxicity despite increased fat mass [12-13].

Liver X receptors (LXR), including two isoforms of $\alpha$ and $\beta$, are members of the nuclear receptor family. Most studies of LXR have been performed in non-adipose tissue, showing a positive role of LXR in central metabolic pathway regulation including cholesterol, glucose and lipid homeostasis; however, results in recent years suggest that LXR may have important modulatory roles in adipose tissue [14-15]. However, studies have reported inconsistent or even contradictory results with regard to the role of LXR in regulating adipogenesis, lipogenesis and insulin sensitivity [16-17]. Recently, Beaven et al [18] have shown that loss of LXR impairs hepatic lipogenesis, accompanied by a reciprocal increase in adipose lipid storage, by promoting adipose PPAR $\gamma$ 
pathway activity, indicating possible cross-talk between LXR and PPAR $\gamma$ in adipose tissue. Cross-talk between LXR and PPAR $\gamma$ in adipose tissue is also supported by our previous study that showed an antagonizing effect of LXR on PPAR $\gamma$ in the regulation of adiponectin expression [19].

Activation of PPAR $\gamma$ in adipose tissue by thiazolidinedione ligands promotes adipose lipid storage and secondarily increases insulin sensitivity in liver and muscle [20]. However, the role of LXR in the alteration of adipose expansion and its relation to insulin resistance has not been studied. Furthermore, whether the possible interference of the PPAR $\gamma$ pathway in adipose tissue by LXR activation results in impairment of adipose expansion and thus impairs insulin sensitivity needs to be clarified in order to comprehensively understand the net outcome of the advantageous and disadvantageous effects of LXR activation.

\section{Results}

LXR activation reduces fat mass accompanied by hepatomegaly and hypertriglyceridemia in mice fed on HFD

To determine the consequence of LXR activation in obesity, C57/BL6 mice were fed a high-fat diet for 12 weeks to induce obesity and subsequently treated for 3 weeks with dual LXR $\alpha / \beta$ agonist T0901317. Fig. 1a shows growth curves of C57/BL6 mice fed a high-fat diet or ND over a 15 week period. Mice on a high-fat diet had higher body weight than those on a ND from 8 weeks (week 8: ND=26.1 $\pm 0.7 \mathrm{~g}$; $\mathrm{HFD}=28.5 \pm 0.6 \mathrm{~g}$; HFD+T0901317=28.7 $\pm 0.8 \mathrm{~g}, \mathrm{P}<0.05)$, whereas the body weight of HFD mice in the presence or absence of 3 weeks of T0901317 (30 mg/kg per day) treatment was comparable (week 15: HFD=31.4 \pm 0.7 g; HFD+T0901317=31 $\pm 0.8 \mathrm{~g}, \mathrm{P}>0.05$, Fig. 1a). Consistent with the body weight, the food intake between them was also similar (Fig. 1b). Unexpectedly, T0901317 administration significantly decreased the fat mass of the epididymal (Fig. 1c), peri-renal and inguinal tissue, whereas liver weight in T0901317-treated HFD mice was doubled than that of the liver in HFD mice with pale appearance (Fig. 1d and Fig. 1e). In the histological analysis, the adipocyte size was significantly reduced in the T0901317-treated HFD mice (Fig. 1f and Fig. 1g). In consistent to the enlarged liver, histological staining with H\&E (Fig. 1h) and red oil (Fig. 1i) confirmed substantial lipid accumulations in the livers of T0901317-treated HFD mice. The serum TG level was significantly higher (Fig. 1j); whereas adipokines of adiponectin and leptin secretion into the circulation were significantly lower in T0901317-treated HFD mice than those in HFD mice (Fig. 1k and Fig. 11). 


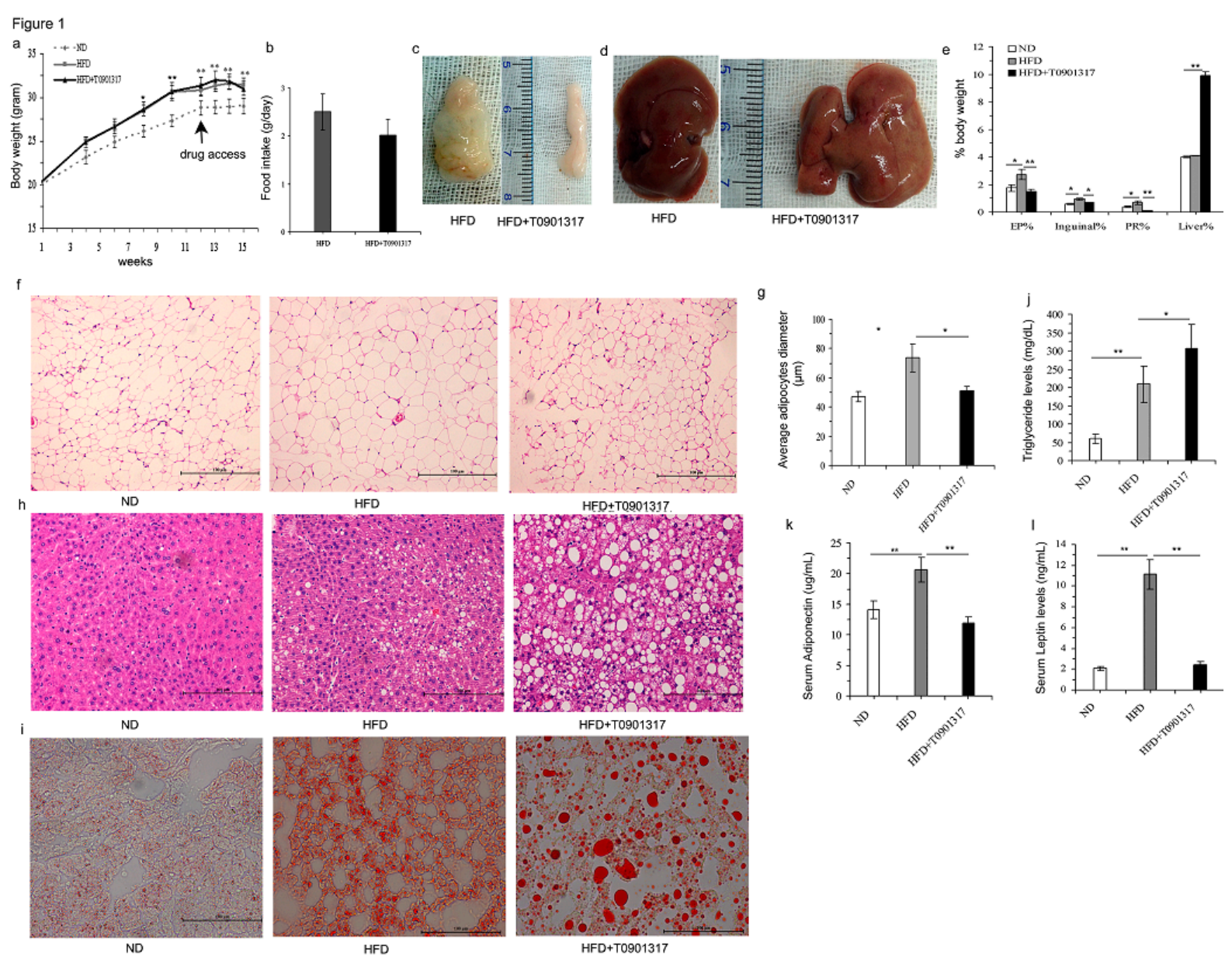

Fig. 1 Characterization of T0901317-treated mice on HFD

(a) Mice on ND, HFD with vehicle (HFD) treatment and high-fat diet with T0901317 (30 mg/kg. I.P. treatment; HFD+T0901317) were weighed weekly (n=12 in each group). ${ }^{*} P<0.05$ vs mice on ND. (b) Food intake in HFD mice with or without T0901317 treatment (n=10-12 in each group). (c) Gross morphology of epididymal fat and (d) liver from HFD mice with or without T0901317 treatment. (e) At 15 weeks of age ( 3 weeks of vehicle or T0901317 treatment), mice were fasted for $12 \mathrm{~h}$, sacrificed and organs harvested. White adipose tissue pads [including epididymal (EP), inguinal and peri-renal (PR) fat mass] and liver weight are shown normalised to total body weight ( $\mathrm{n}=10-12$ in each group). (f) Representative H\&E sections of epididymal fat from mice of different groups. Magnification=100×. (g) Average adipocyte diameter of epididymal fat from mice of different groups ( $\mathrm{n}=8-12$ in each group). (h) Representative H\&E sections of liver from mice of different groups. Magnification=100×. (i) Representative frozen sections of liver stained with Red-Oil from mice of different groups. Magnification=100×.(j) TG levels, (k) Adiponectin levels and (l) leptin levels in serum collected from mice of different groups (n=8-12 in each group).Values are mean $\pm \mathrm{SEM} ;{ }^{*} P<0.05$ and ${ }^{* *} P<0.01$

\section{LXR activation promotes adipocyte apoptosis and inflammation in epididymal fat}

In order to clarify whether the morphological changes in adipose tissue of T0901317-treated mice were associated with decreased cell numbers, apoptotic cells in the adipose tissue of epididymal fat were assessed. There was no difference in apoptotic nuclei numbers in epididymal fat between mice on ND and HFD with vehicle treatment; however, increased apoptosis was indicated by a 3.5 -fold increase in TUNEL 
immunoreactivity in nuclei in epididymal fat of T0901317-treated HFD mice as compared with that in vehicle HFD mice (Fig. 2a and Fig. 2b). Consistently, protein levels of cleaved caspase 9, caspase 3, and PARP, typical molecules of the apoptotic pathway, were all increased by T0901317 treatment (Fig. 2c and Fig. 2d). As seen in Fig.2e and Fig. 2f, HFD induced macrophage infiltration into epididymal fat revealed by F4/80 staining and expression levels, which was further exacerbated by T0901317 treatment. In addition, the expression levels of MCP-1, TNF- $\alpha$ and IL-6 were all significantly increased by T0901317 treatment, consistent with the inflammatory effect of adipocyte death. Picrosirius staining viewed under visible light suggested increase in total collagen in epididymal fat of HFD mice, consistent with increased Col6-1a and MMP-2 and MMP-9 expressions. However, there was no difference in total collagen deposition or expressions (except MMP-2) between HFD mice with or without treatment T0901317 (Fig. 2g and Fig. 2h).

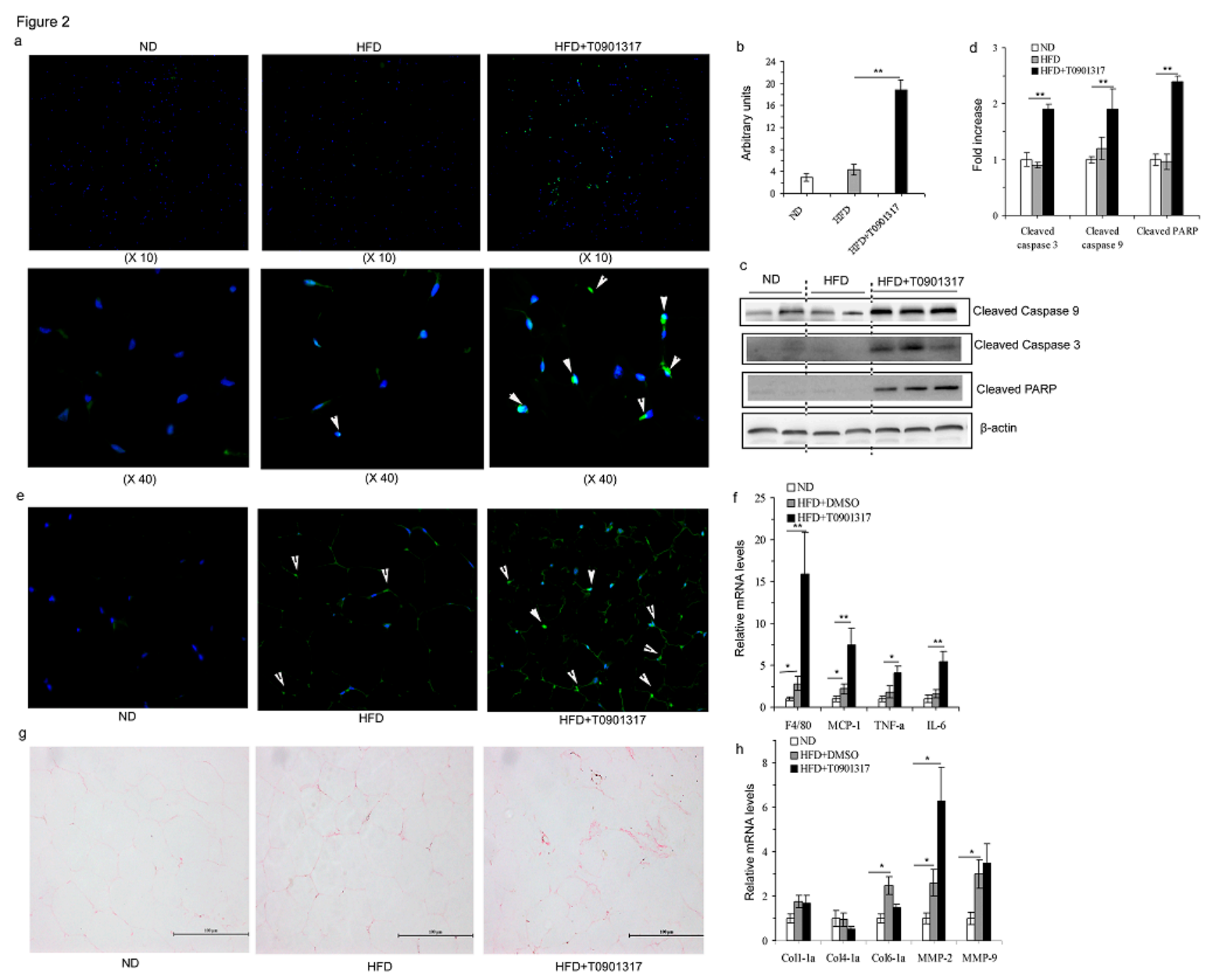

Fig. 2 Adipocyte apoptosis and inflammation in T0901317-treated mice on HFD

(a) Epididymal fat tissue sections of mice on ND, HFD with vehicle (HFD) treatment and HFD with T0901317 (30 mg $/ \mathrm{kg}$ I.P. treatment; HFD+T0901317) were immunostained to characterise apoptotic nuclei TUNEL ( $\mathrm{n}=5-6$ in each group). Normal nuclei are stained with DAPI and apoptotic nuclei are stained in green. Green apoptotic nuclei are indicated by arrows. Magnification=100×/400×. (b) Quantification of TUNEL-positive cells in epididymal fat from mice of different groups ( $n=5-6$ in each group). (c) Protein levels of cleaved caspase-9, 3 and 
PARP in epididymal fat detected by western blotting from mice of different groups. (d) Quantification of above protein levels ( $\mathrm{n}=5-6$ in each group). (e) Immunohistochemistry staining of F4/80 in epididymal fat from mice of different groups. F4/80 positive adipocytes are indicated by arrows. Magnification=200×. (f) Quantification of relative expression of inflammatory genes by Realtime-PCR analysis in epididymal fat from mice of different groups ( $\mathrm{n}=5-6$ in each group). (g) Representative picrosirius staining viewed under visible light from mice of different groups. Magnification=200×. (h) Quantification of relative expression of collagen and MMPs genes by Realtime-PCR analysis in epididymal fat from mice of different groups ( $\mathrm{n}=5-6$ in each group). Values are mean $\pm \mathrm{SEM} ; * P<0.05$ and $* * P<0.01$.

\section{LXR activation increases lipolysis and inhibits PPARy mediated transcriptional activity}

To better understand the mechanism by which T0901317 treatment decreases fat mass and causes hyperlipidemia, we next studied the basal lipolysis, as well as insulin induced anti-lipolytic action. In addition to HFD, fasting FFA levels in circulation was further increased by T0901317 administration in HFD-mice (Fig. 3a). Perilipin1 (PLIN1), the most abundant lipid droplet-coating protein blocking basal lipolysis in adipocytes, was consistently decreased in epididymal fat of HFD mice, with the lowest level in T0901317-treated HFD mice (Fig. $3 \mathrm{~b}$ and Fig. 3c). Furthermore, mice on ND exhibited a nearly 35\% drop in fasting FFA, reflecting insulin's ability to inhibit lipolysis. However, the FFA suppression to exogenous insulin was blunted in vehicle-treated HFD mice $(P=0.076)$, which was further impaired by T0901317 administration (Fig. 3a). Insulin is known to suppress lipolysis by inactivating HSL; we thus investigated the phosphorylated forms of HSL levels in epididymal fat in HFD mice administrated with insulin. Indeed, HSL phosphorylation (s660) levels (but not s563 and s565) and its upstream protein of ATGL were significantly higher in epididymal fat of T0901317-treated HFD mice (Fig. 3d and Fig. 3e).

We next measured the mRNA levels of several genes involved in lipogenesis in epididymal fat. As expected, HFD increased expression of the genes SREBP-1c and Fasn, instead of PPAR $\gamma$, ACC and PCG- $1 \alpha$ in epididymal fat. Surprisingly, T0901317 treatment further induced the expression of SREBP-1c, Fasn and PPAR $\gamma$ without affecting ACC and PCG-1 $\alpha$ despite the fat mass reduction in HFD mice (Fig. 3f).

PPAR $\gamma$ is known to be a powerful promoter of lipogenesis and adipogenesis in adipose tissue, and it plays a critical role in adipose expansion. Based on the contrary pattern of PPAR $\gamma$ and fat mass reduction, we next investigated the effect of T0901317 administration on PPAR $\gamma$ target genes. As shown in Fig. 3g, in epididymal fat, HFD induced increases in PPAR $\gamma$ typical target gene expression, including expression of the lipogenic genes aP2, LPL (the gene responsible for hydrolysis of lipoprotein-bound triglycerides supplying fatty acids and glycerol to adipose depot) and FAT/CD36 (the gene responsible for fatty acid transport to adipose tissue), but reduced expression of the insulin-sensing gene adiponectin .Surprisingly, all the above mentioned PPAR $\gamma$ target genes were decreased by T0901317 treatment in HFD mice, suggesting an antagonizing effect of LXR on the 
PPAR $\gamma$-mediated transcriptional activity, and this finding was supported in vitro study. Fig. $3 \mathrm{~h}$ showed that the binding of PPAR $\gamma$ to PPRE was increased with PPAR $\gamma$ agonst of pioglitazone treatment, whereas T0901317 treatment decreased the activity in presence or absence of pioglitazone in 3T3-L1 adipocytes analyzed by electrophoretic mobility shift assays (EMSA).

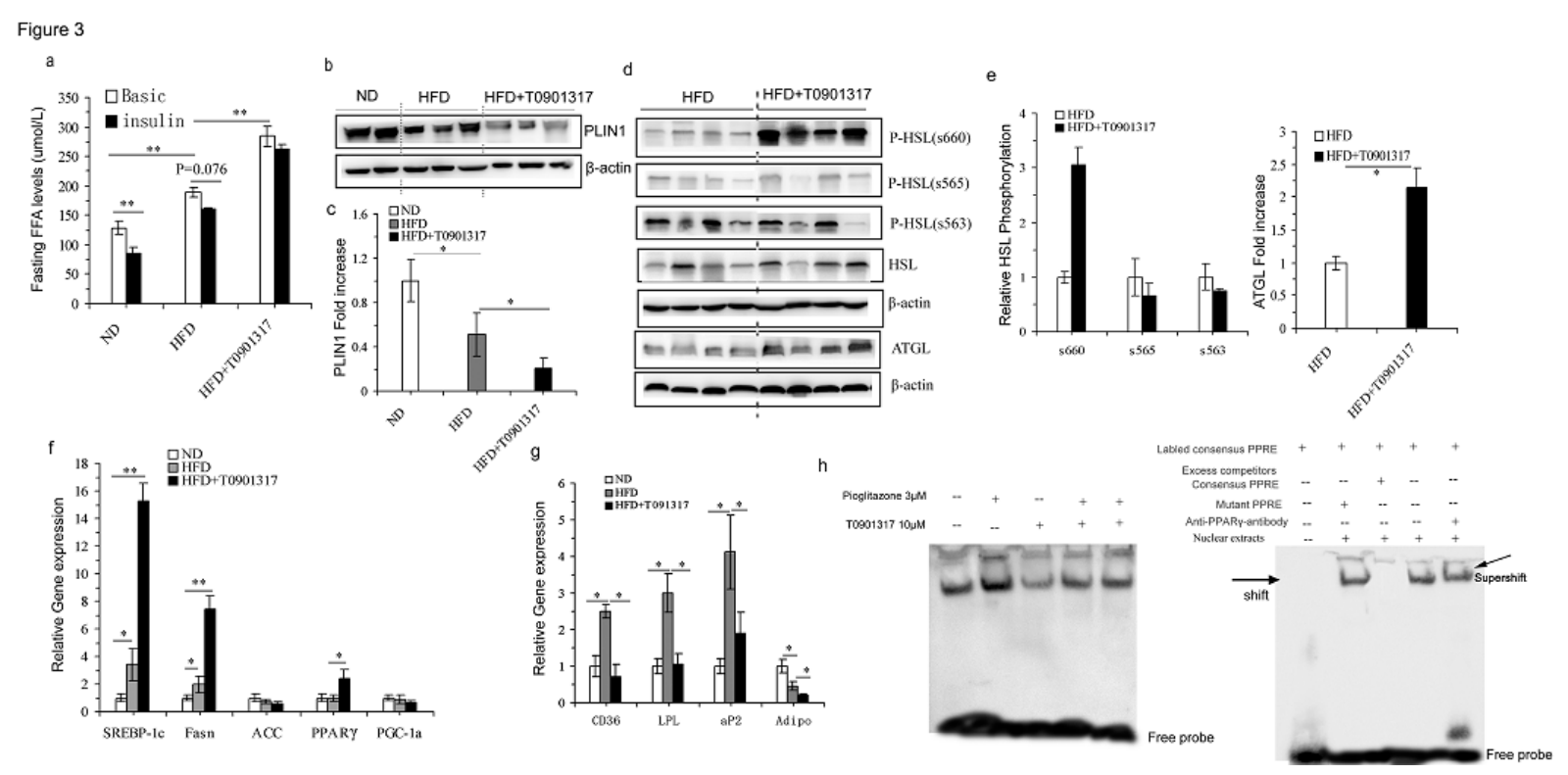

Fig. 3 Lipolysis in T0901317-treated mice on HFD and the effect of LXR on PPARy transcriptional activtity

(a) Serum FFA levels in mice on ND, HFD with vehicle (HFD) treatment and HFD with T0901317 (30 mg/kg I.P. treatment; HFD+T0901317) after 10 minutes of saline (as basic) or insulin (10 IU/kg I.P.) injection before sacrifice ( $\mathrm{n}=5-6$ in each group). (b) Protein levels of PLIN1 in epididymal fat detected by western blotting from mice of different groups. (c) Quantification of PLIN1 protein levels ( $\mathrm{n}=4$ in each group). (d) Lipolytic proteins of ATGL and phosphorylation forms of HSL detected by western blotting from epididymal fat extracts of mice pre-treated with insulin before sacrifice. (e) Quantification of ATGL and relative phosphorylated HSL levels (n=4 in each group). (f) Quantification of relative expression of lipogenic genes by Realtime-PCR analysis in epididymal fat from mice of different groups ( $\mathrm{n}=5-6$ in each group). (g) Quantification of relative expression of PPAR $\gamma$ target genes by Realtime-PCR analysis in epididymal fat from mice of different groups ( $\mathrm{n}=5-6$ in each group). (h) The binding of PPAR $\gamma$ to PPRE in the presence or absence of pioglitazone or T0901317 in differentiated 3T3-L1 adipocytes in EMSA analysis. Values are mean $\pm \mathrm{SEM}, * P<0.05$ and $* * P<0.01$.

\section{LXR activation induces lipid accumulation in the liver}

Generally, increased lipolysis and FFA secretion from adipose tissue leads to ectopic fat accumulation, and which was indeed found in T0901317 treated HFD mice showing enlarged liver and lipid accumulation (Fig. 1d, $1 \mathrm{~h}$ and 1i). Consistent with lipid deposition, liver lipogenic genes of SREBP-1c and Fasn were significantly increased. In contrast to epididymal fat, the LPL gene, responsible for hydrolysis of lipoprotein-bound triglycerides supplying fatty acids for liver, was significantly induced by T0901317 administration. By contrast, CD36, the gene for fatty acid transport, was not changed by T0901317 treatment (Fig. 4a), suggesting that a 
decreased fatty acid influx in adipose tissue might facilitate more fatty acid flow to the liver resulting in triglyceride synthesis. Furthermore, genes responsible for fatty acid oxidation including CPT1 $\alpha$ and PPAR $\alpha$ were decreased, supporting lipid deposition in the liver (Fig. 4b).

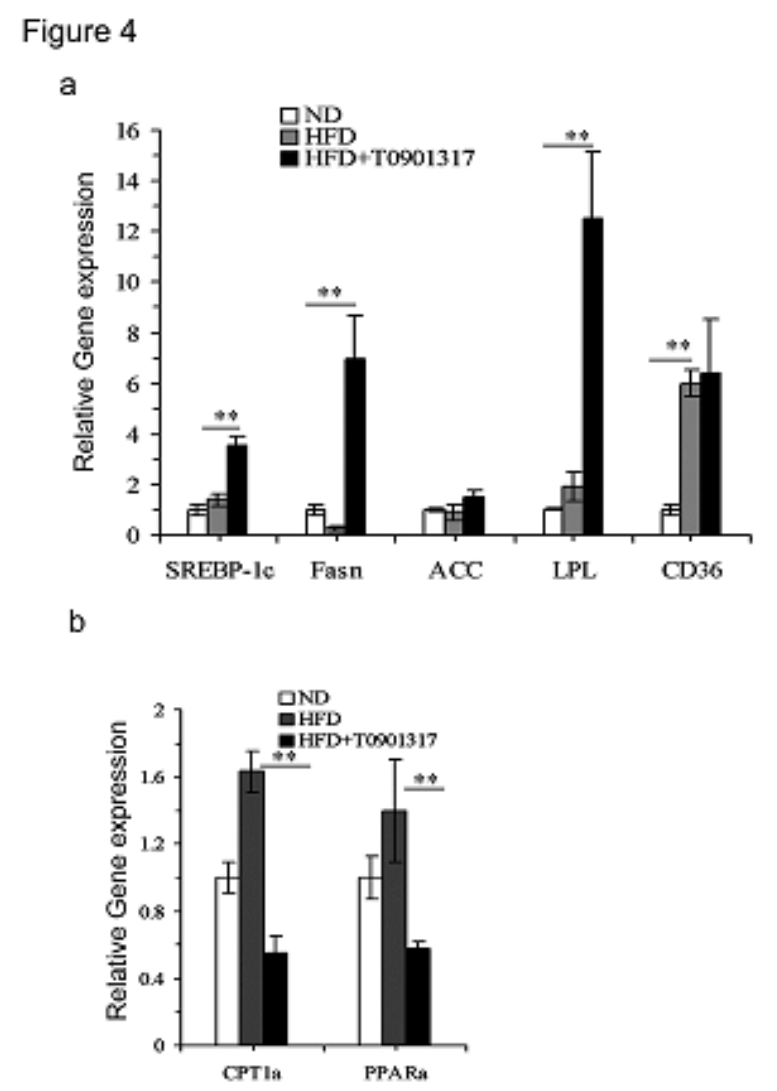

\section{Fig. 4 Lipid deposition related genes in the liver of T0901317-treated mice on HFD}

(a) Quantification of the relative expression of lipogenic genes by Realtime-PCR analysis in liver from mice of different groups ( $\mathrm{n}=5-6$ in each group). (b) Quantification of the relative expression of fatty acid oxidative genes by Realtime-PCR analysis in liver from mice of different groups ( $\mathrm{n}=5-6$ in each group). Values are mean $\pm \mathrm{SEM}, * P<0.05$ and $* * P<0.01$.

\section{LXR activation induces insulin resistance in epididymal fat and in the whole body}

The change of morphology and metabolism of adipose tissue is usually associated with impaired glucose utilisation in adipose tissue, leading to impaired glucose homeostasis in the whole body; therefore, we studied whether LXR affected the glucose response and insulin sensitivity in T090131- treated HFD mice.

Fifteen weeks of HFD successfully induced diabetes and insulin resistance in C57/BL6 mice; these mice showed a significantly higher glucose and insulin response in IPGTT than mice on ND (Fig. 5a-d). Although the glucose response was comparable in IPGTT between T0901317-treated and vehicle-treated HFD mice (Fig. 5a and Fig. 5b), the insulin response of T0901317-treated mice was significantly higher than that of vehicle-treated mice, implicating a decrease in insulin sensitivity with T0901317 treatment (Fig. 5c and Fig. 5d). The decreased 
insulin function was further confirmed by ITT. As shown in Fig. 5e and Fig. 5f, the area under curve of glucose was significantly higher in T0901317-treated HFD mice after insulin overloading, indicating decreased whole-body insulin action by LXR activation.

To further clarify the effect of LXR on glucose uptake in adipose tissue, we studied the glucose utilization in vitro. In differentiated 3T3-L1 adipocytes, both 1 and $10 \mu \mathrm{M}$ T0901317 treatments decreased basal glucose uptake, as well as diminished the glucose uptake increase stimulated by $100 \mathrm{nM}$ insulin (Fig. 5g).

In accord with these results, the typical markers of insulin signalling activity, Akt phosphorylation and Glut-4 protein translocation to the membrane after insulin loading, were markedly decreased in EP fat of T0901317-treated mice (Fig. 5h and Fig. 5i). Consistent with increased insulin levels, histological analysis revealed enlarged and an increased number of pancreatic islets in T0901317-treated mice (Fig. 5j and Fig. 5k).

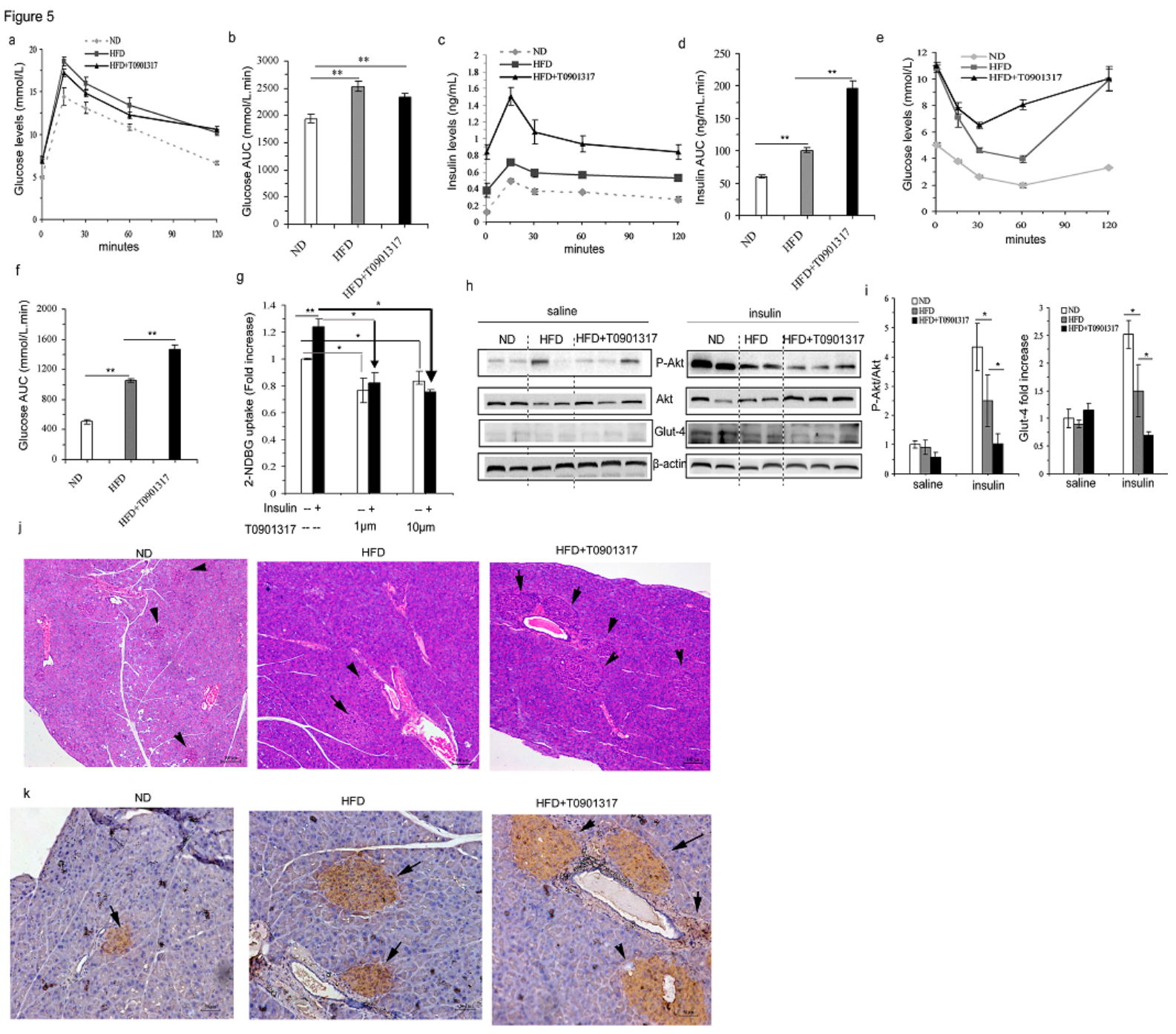

Fig. 5 T0901317-treated mice are glucose tolerant but insulin resistant

(a) Glucose responses in IPGTT and (b) glucose area under curve in IPGTT of mice on ND, HFD and HFD with T0901317 administration after overnight fast ( $\mathrm{n}=5-6$ in each group). (c) Time course measurements of insulin from the same IPGTT and (d) insulin 
area under curve in IPGTT ( $\mathrm{n}=5-6$ in each group). (e) Glucose responses in ITT and (f) glucose area under curve in ITT in mice from different groups after fasted for $4 \mathrm{hr}$ (n=5-6 in each group). (g) Differentiated 3T3-L1 was treated by different doses of T0901317 in the presence or absence of $100 \mathrm{nM}$ insulin, and basal and insulin-stimulated 2-NDBG were measured. Fluorescence intensity of 2-NBDG was recorded by absorbance measurements. Values are shown as mean $\pm \mathrm{SD}, \mathrm{n}=3 \mathrm{C}$. (h) Phosphorylation of Akt and membrane GLUT-4 translocation were detected by western blotting from mice of different groups pre-treated with saline or insulin before sacrifice. (i) Quantification of relative above protein levels ( $n=4$ in each group). (j) Representative H\&E sections of pancreatic islets from mice of different groups. Magnification=200×. (k) Representative immunohistochemistry sections of insulin-positive pancreatic islet staining from mice of different groups. Magnification=200×.

\section{Discussion}

The present study demonstrates that LXR activation causes a reduction in adipose tissue mass but results in massive fatty livers, accompanied by increased adipocyte apoptosis and lipolysis, as well as decreased PPAR $\gamma$ mediated transcriptional activity in adipose tissue. Moreover, impaired adipose expansion by LXR activation is associated with decreased insulin signalling in adipose tissue and decreased insulin sensitivity of the whole body in HFD-fed mice.

Consistent with the findings of Beaven et al and Korach-André and co-workers [18,21] who described that global LXR deletion in the setting of obesity shifts the programme of de novo lipogenesis from the liver to adipose tissue, we found an opposite phenotype, showing that LXR activation caused reductions in fat pads but liver enlargement and fatty steatosis. Loss of adipocytes through apoptosis by LXR activation might be an important process leading to fat mass reduction in the present study. Apoptosis of adipose tissue is relatively poorly studied compared to that in other tissues; yet increased adipocyte apoptosis has been recently proposed to contribute to obesity, and to differences in regional fat distribution or expansion and insulin resistance, in both obese animals and humans [9,22-23]. As shown in the present study and others [9,22-23], increased adipocyte apoptosis usually results in macrophage infiltration and inflammation, which are associated with insulin resistance. Furthermore, insulin resistance induced by inflammatory factors is reversed by interference with apoptosis initiation via CASP3/7 inhibition.

Lipid storage is determined by the balance between fat synthesis (lipogenesis) and fat breakdown (lipolysis/fatty acid oxidation). Lipogenesis encompasses the processes of fatty acid synthesis and triglyceride synthesis, and takes place in both the liver and in adipose tissue, and it is the crucial process for adipose expansion. Although LXR agonists are well-characterised inducers of hepatic lipogenesis via SREBP-1c and its response genes FAS, ACC and SCD1 [24-25], we did not find a role of LXR activation in lipogenesis in adipocytes, although significantly increased expression of SREBP-1c and FAS were found in epididymal fat. 
This finding is supported by Archer et al [26] who showed increased SREBP-1c expression in visceral fat but lower visceral fat mass. These results suggest that at least in rodent adipose tissue, LXR and SREBP-1c are not the primary regulator of lipogenesis; this conclusion is supported by data from SREBP-1c ${ }^{-/-}$mice and mature adipocytes isolated from $\mathrm{LXR} \alpha / \beta^{-/-}$mice, both of which showed a similar level of lipogenesis to that in their wild-type littermates [27].

However, increased basal lipolysis supported by increased fasting NEFA levels in the circulation by T0901317 treatment might contribute to reduced adipocyte size and fat mass by LXR activation. Similar treatment with GW3965 resulted in smaller fat cells [26], indicative of increased triglyceride utilisation. An effect of LXR on basal lipolysis is also supported by findings in human adipocytes and adipose tissue LXR $\alpha$ knockout (ATaKO) mice [28-29]. Down-regulation of lipid droplet-coating proteins of PLIN1, as found in our present study might be the molecular mechanism, for low levels or absence of PLIN1 has been implicated in enhanced spontaneous lipolysis both in mice and humans [30-32]. In addition, we found that the insulin induced anti-lipolytic action was significantly blunted by LXR activation. Insulin is known to suppress lipolysis by inactivating HSL [33]. In T0901317-treated HFD mice, we did find HSL phosphorylation and its upstream molecular of ATGL were higher than those in vehicle-treated HFD mice with same dose of insulin administration, supporting the reduction of fat mass was associated with lipolysis. Furthermore, expression levels of CD36 and LPL (proteins involved in lipid clearance from the circulation and deposition in adipose tissue as triglycerides) were decreased, which might contribute to increased TG and NEFA levels in the circulation and decreased fat mass in T0901317-treated HFD mice. PPAR $\gamma$ is critically required for adipose tissue expansion by increasing lipogenesis and adipocyte proliferation. Indeed, consistent with reductions in fat mass and increases in adipocyte apoptosis, we found that PPAR $\gamma$ mediated transcriptional acitivity was inhibited by LXR, which was supported by the reduced PPAR $\gamma$-PPRE binding activity in T0901317-treated 3T3-L1 cell, as well as the inhibited PPAR $\gamma$ target genes expression in epididymal fat in T0901317-treated HFD mice. Although the mechanism of LXR interfering with the PPAR $\gamma$ pathway was not further studied, the fact that LXR shares its heterodimerising partner (that is RXR) with PPARs and sometimes competes for the same DNA response elements suggests that LXR could affect PPAR signalling [34-35].

Impaired adipose expansion was generally associated with ectopic lipid accumulation and insulin resistance. Indeed in the present study, moderate hepatomegaly and steatosis, as well as higher insulin levels, decreased insulin sensitivity and enlarged pancreatic islets were consistent with other animal models of impaired adipose expansion and human lipoatrophy or lipodystrophy [36-37]. The role of LXR activation in fatty acid synthesis in 
the liver is well established [38-39]. Indeed, our study also found that typical genes of lipogenesis, including SREBP-1c and FAS, were significantly induced by T0901317 administration. Importantly, we further found increased LPL and decreased CTP1 $\alpha$ and PPAR $\alpha$ in the liver, suggesting an increased capacity of fatty acid intake and decreased fatty acid oxidation in the liver, which might also be another mechanism for hepatic steatosis by LXR activation. It is worth pointing out that pancreatic islets in T0901317-treated mice generated double the insulin levels, which at least suggests insulin resistance instead of a direct stimulating effect of LXR activation, for blood glucose responses in T0901317-treated mice were comparable instead of lower than those in HFD mice, and this finding was supported by ITT results which showed that T0901317-treated mice had a blunted glucose response. Decreased insulin efficiency was most likely associated with increased NEFA levels and decreased levels of the insulin-sensitising adipokines adiponectin and leptin.

Glucose uptake in adipose tissue impacts on whole-body glucose homeostasis. The effect of T0901317 on glucose utilisation in adipocyte is not consistent [40-42]. In our study, we found that T0901317 decreased both basal and insulin-stimulated glucose uptake in 3T3-L1 cells. Decreased glucose uptake in adipose tissue was supported by insulin-induced Akt activity and Glut-4 membrane translocation in T0901317-treated mice. This result is consistent with the in vitro findings of other studies either using the same cell model or in primary human adipocytes [40,42]. While other studies have shown unchanged or increased basal glucose uptake, the discrepancies between these studies could result from differences in agonists, in vitro cell models and treatment conditions. Despite decreased glucose uptake in adipocytes, whole-body glucose homeostasis was not changed in T0901317-treated mice. This finding probably resulted from the compensatory increase in insulin secretion and pancreatic islet enlargement. The relatively stable glucose metabolism might also be due to decreased gluconeogenesis by LXR activation [41,43-44]. A few studies addressing the role of LXR in carbohydrate metabolism have shown improved glucose tolerance by LXR activation in vivo; however, no study has investigated the fat mass change. No change of systemic glucose homeostasis was shown in ob/ob female mice with T0901317 treatment in the study by Archer et al, in which they also found decreased visceral fat mass with GW3965 treatment [26]. Thus, the adipose tissue loss in these studies might be the reason for the discrepancies. Decreased adipose mass is possibly only caused by a relatively higher dose or longer treatment period, as in our study and the study of Archer et al.

Taken together, the integrated effect of LXR activation is dependent on the action of many organs, particularly adipose tissue and liver. The positive actions of LXR activation on reverse cholesterol transport and hepatic gluconeogenesis could be counteracted by impaired fat storage capacity, increased fat accumulation in 
the liver and aberrant PPAR $\gamma$ signalling in adipose tissue, leading to unexpected metabolic consequences when administering LXR activators in vivo.

\section{Methods and Materials}

\section{Reagents}

The dual LXR $\alpha / \beta$ agonist T0901317 was purchased from Cayman Chemical Company (Ann Arbor, MI, USA) and pioglitazone was purchased from Sigma-Aldrich (Saint Louis, MO, USA). In animal experiments, T0901317 was solubilised in a vehicle containing 3\% DMSO in PBS and administered by i.p. injection at a dose of $30 \mathrm{mg} / \mathrm{kg}$ body weight. In cellular experiments, T0901317 was prepared in a solution of 1:1 DMSO: PBS at a concentration of $1 \mathrm{mM}$, and pioglitazone was solubilised in DMSO at a concentration of $20 \mathrm{mM}$ for further dilution with cell medium.

\section{Animals}

All animal care procedures and methods were approved by the Animal Care Committee of Zhejiang University (China, Protocol 2014_0108). C57BL/6 mice (aged 8 weeks) were purchased from Slack Experimental Animal Center of the Chinese Academy of Sciences (Shanghai, China). A cohort of 36 mice were housed singly and maintained on a $12 \mathrm{hr}$ light-dark cycle. After 1 week of habituation fed on regular chow diet (normal diet [ND], carbohydrate, 63.92\%; protein, 26.18\%; fat, 9.9\%), animals were weighed and divided into two groups: the high-fat diet (HFD) group ( $\mathrm{n}=24)$ was fed a HFD (35\% carbohydrate, $20 \%$ protein and $45 \%$ fat) and the ND control group $(n=12)$.

After 12 weeks, the HFD group was further randomised into two groups of 12 mice. HFD mice were treated for 3 weeks with $30 \mathrm{mg} / \mathrm{kg}$ T0901317 per day $(\mathrm{n}=12)$ or the vehicle $(3 \%$ DMSO in PBS, $\mathrm{n}=12)$ by i.p injection. The ND control group also received the same vehicle treatment by i.p injection. Body weight was recorded once a week and food intake was monitored every day.

\section{Culture and differentiation of 3T3-L1 cells}

3T3-L1 cells were purchased from the American Type Culture Collection (ATCC, Rockefeller, MD,USA) and were cultured in Dulbecco's modified Eagle's medium (DMEM) containing 10\% FBS (Bio-rad, Hercules, $\mathrm{CA}, \mathrm{USA}$ ) and $100 \mathrm{IU} / \mathrm{ml}$ penicillin/streptomycin at $37^{\circ} \mathrm{C}$ in an atmosphere of $5 \% \mathrm{CO} 2$ and $95 \%$ humidity. Two days post-confluent cells (designated as Day 0) were induced to differentiate into adipocytes by the addition of differentiation mixture with DMEM containing $10 \% \quad$ FBS, $10 \mu \mathrm{g} / \mathrm{mL}$ insulin, $\quad 0.5 \quad \mathrm{mM}$ 
3-isobutyl-1-methylxanthine (IBMX) and $1 \mu \mathrm{M}$ dexamethasone. Two days later, culture medium was changed to DMEM supplemented with $10 \%$ FBS and $10 \mu \mathrm{g} / \mathrm{mL}$ insulin for 2 days. The medium was then replaced every other day with DMEM containing 10\% FBS for different periods until day 10 .

\section{In vivo glucose homeostasis assays}

After 3 weeks of treatment, intraperitoneal glucose tolerance tests (IPGTT) and intraperitoneal insulin tolerance tests (ITT) were carried out. Half of the mice ( $n=5-6$ per group) were injected i.p. with glucose (1.5 $\mathrm{g} / \mathrm{kg}$ body weight) following an overnight fast and blood glucose levels were tested from tail blood using One Touch Ultra glucose strips (LifeScan, PA, USA) at 0 (basal), 15, 30, 60 and 120 min. Tail blood was also sampled at each time-point for insulin measurements. The remaining mice ( $n=5-6$ per group) were subjected to ITT. Mice were fasted for $4 \mathrm{hr}$ prior to i.p. injection of insulin at a dose of $0.5 \mathrm{IU} / \mathrm{kg}$ body weight. Blood samples were collected from the tail at 0,15, 30,60 and $120 \mathrm{~min}$, and glucose levels were measured immediately by One Touch Ultra glucose strips (LifeScan).

After these tests, all mice were fasted for $16 \mathrm{hr}$ and injected i.p. with saline ( $\mathrm{n}=5-6$ per group) or insulin (10 U/kg body weight, $n=5-6$ per group) and sacrificed 10 min later by cervical dislocation. Blood samples were obtained and serum was collected and stored at $-80^{\circ} \mathrm{C}$ immediately. Liver, epididymal, peri-renal and inguinal fat pads were carefully excised and weighed, and the pancreas was also dissected. After rinsing in pre-cooled PBS, part of the tissue was placed in storage tubes in a dry ice bath until the end of experiment, and then stored at $-80^{\circ} \mathrm{C}$ for later protein, RNA extraction and preparation of frozen sections, while the other part was fixed in $4 \%$ formaldehyde for routine pathological staining.

\section{Blood sample assays}

Serum insulin concentrations were determined using the insulin (mouse) EIA kit (Millipore, Billerica, MA, USA). Adiponectin levels in the fasted serum were measured with the adiponectin (murine) EIA kit (Millipore). NEFA, leptin and triglyceride levels in the fasted serum were measured with the FFA assay kit (Cayman Chemical), mouse leptin ELISA kit (Millipore) and mouse TG quantification colormetric kit (Biovision, San Francisco, CA, USA). All kits were used according to the manufacturer's protocols. 


\section{Histological analysis of adipose tissue and liver}

Adipose, liver and pancreatic tissues fixed in $4 \%$ formaldehyde were then embedded in OCT compound and cut into sections (thickness, $4 \mu \mathrm{m}$ ) according to a standard protocol. The sections were stained with hematoxylin and eosin (H\&E) and examined under a light microscope. Size of adipocytes was analysed using Image Pro-plus 6.0 software on H\&E staining sections. Frozen liver sections (thickness, $5 \mu \mathrm{m}$ ) were stained with Oil Red O to assess hepatic lipid content. The extent of adipocyte apoptosis in epididymal fat was determined using a TUNEL kit (Roche Diagnostics, Indianapolis, IN, USA). To quantify apoptosis, 10 visual fields at $400 \times$ from each section were analysed for TUNEL-positive cells. A TUNEL index was determined using the following formula: (number of stained cells/total number of cells) $\times 100$. Immunohistochemical staining was carried out to determine the levels of F4/80 in epididymal fat sections (rabbit anti-mouse; 1:50; Invitrogen, Grand Island, NY, USA) and insulin (rabbit anti-mouse, 1:200; Santa Cruz, Dallas, TX, USA) in pancreatic tissue sections using standard protocols. A Cy2-conjugated goat anti-rabbit secondary antibody (H\&L, Alexa Fluor®488; Abcam, Cambridge, MA, USA) at a 1:1,000 dilution was used as a secondary antibody for F4/80 detection, and sections were then counterstained with DAPI, cover-slipped and examined under a fluorescence microscope. An anti-rabbit horseradish peroxidase-linked secondary antibody and diaminobenzidine (Beijing Zhongshan Goldenbridge Company, Beijing, China) were used to detect specific binding for insulin, and sections were counterstained with hematoxylin. Picrosirius staining for epididymal fat sections was carried out for collagen with picrosirius red solution (picrosirius $0.4 \mathrm{~g}$ in saturated aqueous solution of picric acid $100 \mathrm{ml}$ ) and vied under light microscopy.

\section{Quantitative real-time RT-PCR}

Total RNA was isolated using TRIzol (Invitrogen, Grand Island, NY, USA) and reverse-transcribed with random hexamers by using TaqMan reverse-transcription reagents kit (Applied Biosystems Inc., San Francisco, CA, USA) according to the manufacturer's protocol. Real-time PCR was performed using the 7500 real-time PCR system (Applied Biosystems) and SYBR Green qPCR Kit (TaKaRa, Dalian, China). Relative expression was normalised to that of GAPDH as an internal control for quantification of individual mRNA species and calculated using the formula $2(-\Delta \Delta \mathrm{Ct})$. Primer sets were listed in Table S1.

\section{Western blotting}

Equal amounts of protein $(50 \mu \mathrm{g})$ denatured by boiling were separated by $10 \%$ SDS-polyacrylamide gel electrophoresis, transferred to an Immun-Blot PVDF membranes (Millipore) and blocked with 5\% non-fat milk 
for $1 \mathrm{hr}$ at room temperature. Membranes were then incubated with primary antibodies (diluted 1:1,000) including anti-rabbit cleaved caspase 3 and 9, Cleaved PARP, phosphorylated HSL (s660, s565 and s563), HSL, ATGL, Perilipin (PLIN1), phosphorylated Akt, Akt, Glut-4 (plasma membrane protein) and $\beta$-actin (Cell Signaling Technology, Danvers, MA, USA) at $4^{\circ} \mathrm{C}$ overnight. After incubation with horseradish-peroxidase-conjugated goat anti-rabbit secondary antibodies at room temperature for $1 \mathrm{hr}$, immunoreactive proteins were detected using a chemiluminescent ECL assay kit (Millipore).

\section{Glucose uptake analysis}

Glucose uptake activity was measured using a fluorescent D-glucose analogue 2-[N-(7-nitrobenz-2-oxa-1,3-diaz-ol-4-yl)amino]-2-deoxy-D-glucose (2-NBDG) in differentiated 3T3-L1 cells. Briefly, differentiated 3T3-L1 cells on 12 well plates were treated with different concentrations of T0901317 (0, 1.0 and $10.0 \mu \mathrm{M})$ for $24 \mathrm{~h}$. Then, cells were washed with Dulbecco's Phosphate Buffered Saline (DPBS) and incubated with $100 \mathrm{nM}$ insulin in no-glucose DMEM for $10 \mathrm{~min}$ and $60 \mu \mathrm{M}$ 2-NBDG was added to a medium for another $1 \mathrm{hr}$. The medium was then washed twice with cold DPBS to remove free 2-NBDG. The cells in each well were suspended with DPBS after trypsinization and subsequently transferred to 96 black well fluorescence plates. The fluorescence intensity of cellular 2-NBDG in each well was measured at an excitation wavelength of $485 \mathrm{~nm}$ and an emission wavelength of $535 \mathrm{~nm}$ using a fluorescent microplate reader.

\section{Electrophoretic mobility shift assay}

Differentiated 3T3-L1 cells were treated with DMSO, $10 \mu \mathrm{M}$ T0901317 and $3 \mu \mathrm{M}$ pioglitazone or co-treated with $10 \mu \mathrm{M}$ T0901317 and $3 \mu \mathrm{M}$ pioglitazone for $24 \mathrm{hr}$, and nuclear protein was extracted and quantified. Electrophoretic mobility shift assay(EMSA)was performed using double stranded biotin-labelled oligo probes of PPRE (Beyotime Biotechnology, Haimen, China). PPAR consensus oligo sequences were as follows: 5'- CAA AAC TAG GTC AAA GGT CA -3' and 3'- GTT TTG ATC CAG TTT CCA GT -5'. PPAR mutant oligo sequences were as follows: 5'- CAA AAC TAG CAC AAA GCA CA -3' and 3'- GTT TTG ATC GTG TTT CGT GT -5'. To measure DNA-protein interaction, $1 \mu \mathrm{l}$ of oligo probe was incubated with nuclear extract $(20 \mu \mathrm{g})$, EMSA/Gel-Shift binding buffer and nuclease-free water for $20 \mathrm{~min}$ at room temperature in a total volume of 10 $\mu 1$. Unlabelled oligo probe was used for the cold probe competitive reaction, and unlabelled mutant olig probe was used as the mutation probe for the cold competitive reaction. Anti-rabbit PPAR $\gamma$ antibody (Cell Signaling Technology, Denver, MA, USA) was added for super-shift reaction. The reaction mixture was subjected to 
electrophoresis $(100 \mathrm{~V}$ in $0.5 \times$ Tris-buffered EDTA solution at room temperature) using $8 \%$ nondenaturing polyacrylamide gels, then transferred to a positively charged nylon membrane (Beyotime Biotechnology, Haimen, China), UV cross-linked and blocked. After incubation with $5 \mu$ l streptavidin-HRP conjugate, immunoreactive proteins were detected using a chemiluminescent ECL assay kit (Millipore). Statistical analysis

Data are expressed as means \pm SEM (in vivo studies) or means \pm SD (in vitro studies). Differences between the means of individual groups were analysed with independent t-tests or one-way ANOVA and LSD multiple range tests. Two-way repeated measures were used for comparisons between glucose and insulin levels of IPGTT or ITT using the statistical software package SPSS 16.0. A significant difference was defined as $\mathrm{P}<0.05$. Each in vitro experiment was conducted in triplicate.

Acknowledgments: This work was supported by the National Natural Science Foundation of China (General Program, 8127092, Hong Li) and the National Natural Science Foundation of China (General Program, 81471099, Fenping Zheng) and the Zhejiang Provincial Natural Science Foundation of China, Y17H070008, Fenping Zheng). The received funds can cover the costs to publish in open access.

Author contributions: Fenping Zheng and Hong Li conceived and designed the experiments; Yueting Dong, Zhiye Xu, Ziyi Zhang, Xueyao Yin, Qianqian Pan and Xihua Lin performed the experiments; Fenping Zheng analyzed the data and wrote the paper.

Conflicts of Interest: The authors declare no conflict of interest.

\section{References:}

1. Romacho, T.; Elsen, M.; Rohrborn, D.; Eckel, J. Adipose tissue and its role in organ crosstalk. Acta Physiol (Oxf) 2014, 210, 733-753.

2. Abranches, M.V.; Oliveira, F.C.; Conceicao, L.L.; Peluzio, M.D. Obesity and diabetes: The link between adipose tissue dysfunction and glucose homeostasis. Nutr Res Rev 2015, 28, 121-132.

3. Jankovic, A.; Korac, A.; Buzadzic, B.; Otasevic, V.; Stancic, A.; Daiber, A.; Korac, B. Redox implications in adipose tissue (dys)function-a new look at old acquaintances. Redox Biol 2015, 6, 19-32.

4. Despres, J.P.; Lemieux, I. Abdominal obesity and metabolic syndrome. Nature 2006, 444, 881-887.

5. Lionetti, L.; Mollica, M.P.; Lombardi, A.; Cavaliere, G.; Gifuni, G.; Barletta, A. From chronic overnutrition to insulin resistance: The role of fat-storing capacity and inflammation. Nutr Metab Cardiovasc Dis 2009, 19, 146-152.

6. Murdolo, G.; Bartolini, D.; Tortoioli, C.; Piroddi, M.; Iuliano, L.; Galli, F. Lipokines and oxysterols: Novel adipose-derived lipid hormones linking adipose dysfunction and insulin resistance. Free Radic Biol Med 2013, 65, 811-820.

7. Hirsch, J.; Han, P.W. Cellularity of rat adipose tissue: Effects of growth, starvation, and obesity. $J$ Lipid Res 1969, 10, 77-82.

8. Roncari, D.A.; Lau, D.C.; Kindler, S. Exaggerated replication in culture of adipocyte precursors from massively obese persons. Metabolism 1981, 30, 425-427.

9. Alkhouri, N.; Gornicka, A.; Berk, M.P.; Thapaliya, S.; Dixon, L.J.; Kashyap, S.; Schauer, P.R.; Feldstein, A.E. Adipocyte apoptosis, a link between obesity, insulin resistance, and hepatic steatosis. $J$ Biol Chem 2010, 285, 3428-3438.

10. Medina-Gomez, G.; Gray, S.L.; Yetukuri, L.; Shimomura, K.; Virtue, S.; Campbell, M.; Curtis, R.K.; 
Jimenez-Linan, M.; Blount, M.; Yeo, G.S., et al. Ppar gamma 2 prevents lipotoxicity by controlling adipose tissue expandability and peripheral lipid metabolism. PLoS Genet 2007, 3, e64.

11. Cock, T.A.; Houten, S.M.; Auwerx, J. Peroxisome proliferator-activated receptor-gamma: Too much of a good thing causes harm. EMBO Rep 2004, 5, 142-147.

12. Miles, P.D.; Barak, Y.; He, W.; Evans, R.M.; Olefsky, J.M. Improved insulin-sensitivity in mice heterozygous for ppar-gamma deficiency. J Clin Invest 2000, 105, 287-292.

13. Yamauchi, T.; Kadowaki, T. [the molecular mechanisms by which ppar gamma/rxr inhibitors improve insulin resistance]. Nihon Rinsho 2001, 59, 2245-2254.

14. Laurencikiene, J.; Ryden, M. Liver x receptors and fat cell metabolism. Int J Obes (Lond) 2012, 36, 1494-1502.

15. Nomiyama, T.; Bruemmer, D. Liver $\mathrm{x}$ receptors as therapeutic targets in metabolism and atherosclerosis. Curr Atheroscler Rep 2008, 10, 88-95.

16. Juvet, L.K.; Andresen, S.M.; Schuster, G.U.; Dalen, K.T.; Tobin, K.A.; Hollung, K.; Haugen, F.; Jacinto, S.; Ulven, S.M.; Bamberg, K., et al. On the role of liver x receptors in lipid accumulation in adipocytes. Mol Endocrinol 2003, 17, 172-182.

17. Hummasti, S.; Laffitte, B.A.; Watson, M.A.; Galardi, C.; Chao, L.C.; Ramamurthy, L.; Moore, J.T.; Tontonoz, P. Liver $\mathrm{x}$ receptors are regulators of adipocyte gene expression but not differentiation: Identification of apod as a direct target. J Lipid Res 2004, 45, 616-625.

18. Beaven, S.W.; Matveyenko, A.; Wroblewski, K.; Chao, L.; Wilpitz, D.; Hsu, T.W.; Lentz, J.; Drew, B.; Hevener, A.L.; Tontonoz, P. Reciprocal regulation of hepatic and adipose lipogenesis by liver $\mathrm{X}$ receptors in obesity and insulin resistance. Cell Metab 2013, 18, 106-117.

19. Zheng, F.; Zhang, S.; Lu, W.; Wu, F.; Yin, X.; Yu, D.; Pan, Q.; Li, H. Regulation of insulin resistance and adiponectin signaling in adipose tissue by liver $\mathrm{x}$ receptor activation highlights a cross-talk with ppargamma. PLoS One 2014, 9, e101269.

20. Derosa, G.; Maffioli, P. Peroxisome proliferator-activated receptor-gamma (ppar-gamma) agonists on glycemic control, lipid profile and cardiovascular risk. Curr Mol Pharmacol 2012, 5, 272-281.

21. Korach-Andre, M.; Archer, A.; Gabbi, C.; Barros, R.P.; Pedrelli, M.; Steffensen, K.R.; Pettersson, A.T.; Laurencikiene, J.; Parini, P.; Gustafsson, J.A. Liver $\mathrm{x}$ receptors regulate de novo lipogenesis in a tissue-specific manner in c57bl/6 female mice. Am J Physiol Endocrinol Metab 2011, 301, E210-222.

22. Bennett, G.; Strissel, K.J.; DeFuria, J.; Wang, J.; Wu, D.; Burkly, L.C.; Obin, M.S. Deletion of tnf-like weak inducer of apoptosis (tweak) protects mice from adipose and systemic impacts of severe obesity. Obesity (Silver Spring) 2014, 22, 1485-1494.

23. Tinahones, F.J.; Coin Araguez, L.; Murri, M.; Oliva Olivera, W.; Mayas Torres, M.D.; Barbarroja, N.; Gomez Huelgas, R.; Malagon, M.M.; El Bekay, R. Caspase induction and bcl2 inhibition in human adipose tissue: A potential relationship with insulin signaling alteration. Diabetes Care 2013, 36, 513-521.

24. Schultz, J.R.; Tu, H.; Luk, A.; Repa, J.J.; Medina, J.C.; Li, L.; Schwendner, S.; Wang, S.; Thoolen, M.; Mangelsdorf, D.J., et al. Role of lxrs in control of lipogenesis. Genes Dev 2000, 14, 2831-2838.

25. Yoshikawa, T.; Shimano, H.; Amemiya-Kudo, M.; Yahagi, N.; Hasty, A.H.; Matsuzaka, T.; Okazaki, H.; Tamura, Y.; Iizuka, Y.; Ohashi, K., et al. Identification of liver x receptor-retinoid x receptor as an activator of the sterol regulatory element-binding protein 1c gene promoter. Mol Cell Biol 2001, 21, 2991-3000.

26. Archer, A.; Stolarczyk, E.; Doria, M.L.; Helguero, L.; Domingues, R.; Howard, J.K.; Mode, A.; Korach-Andre, M.; Gustafsson, J.A. Lxr activation by gw3965 alters fat tissue distribution and adipose tissue inflammation in ob/ob female mice. J Lipid Res 2013, 54, 1300-1311.

27. Kalaany, N.Y.; Gauthier, K.C.; Zavacki, A.M.; Mammen, P.P.; Kitazume, T.; Peterson, J.A.; Horton, J.D.; Garry, D.J.; Bianco, A.C.; Mangelsdorf, D.J. Lxrs regulate the balance between fat storage and oxidation. Cell Metab 2005, 1, 231-244.

28. Stenson, B.M.; Ryden, M.; Venteclef, N.; Dahlman, I.; Pettersson, A.M.; Mairal, A.; Astrom, G.; Blomqvist, L.; Wang, V.; Jocken, J.W., et al. Liver x receptor (lxr) regulates human adipocyte lipolysis. J Biol Chem 2011, 286, 370-379.

29. Dib, L.; Bugge, A.; Collins, S. Lxralpha fuels fatty acid-stimulated oxygen consumption in white adipocytes. J Lipid Res 2014, 55, 247-257.

30. Tansey, J.T.; Sztalryd, C.; Gruia-Gray, J.; Roush, D.L.; Zee, J.V.; Gavrilova, O.; Reitman, M.L.; Deng, C.X.; Li, C.; Kimmel, A.R., et al. Perilipin ablation results in a lean mouse with aberrant adipocyte lipolysis, enhanced leptin production, and resistance to diet-induced obesity. Proc Natl Acad Sci US A 2001, 98, 6494-6499.

31. Martinez-Botas, J.; Anderson, J.B.; Tessier, D.; Lapillonne, A.; Chang, B.H.; Quast, M.J.; Gorenstein, D.; Chen, K.H.; Chan, L. Absence of perilipin results in leanness and reverses obesity in lepr(db/db) mice. Nat Genet 2000, 26, 474-479. 
32. Mottagui-Tabar, S.; Ryden, M.; Lofgren, P.; Faulds, G.; Hoffstedt, J.; Brookes, A.J.; Andersson, I.; Arner, P. Evidence for an important role of perilipin in the regulation of human adipocyte lipolysis. Diabetologia 2003, 46, 789-797.

33. Choi, S.M.; Tucker, D.F.; Gross, D.N.; Easton, R.M.; DiPilato, L.M.; Dean, A.S.; Monks, B.R.; Birnbaum, M.J. Insulin regulates adipocyte lipolysis via an akt-independent signaling pathway. Mol Cell Biol 2010, 30, 5009-5020.

34. Ide, T.; Shimano, H.; Yoshikawa, T.; Yahagi, N.; Amemiya-Kudo, M.; Matsuzaka, T.; Nakakuki, M.; Yatoh, S.; Iizuka, Y.; Tomita, S., et al. Cross-talk between peroxisome proliferator-activated receptor (ppar) alpha and liver $\mathrm{x}$ receptor (lxr) in nutritional regulation of fatty acid metabolism. Ii. Lxrs suppress lipid degradation gene promoters through inhibition of ppar signaling. Mol Endocrinol 2003, $17,1255-1267$.

35. Yoshikawa, T.; Ide, T.; Shimano, H.; Yahagi, N.; Amemiya-Kudo, M.; Matsuzaka, T.; Yatoh, S.; Kitamine, T.; Okazaki, H.; Tamura, Y., et al. Cross-talk between peroxisome proliferator-activated receptor (ppar) alpha and liver $\mathrm{x}$ receptor ( $\mathrm{xr}$ ) in nutritional regulation of fatty acid metabolism. I. Ppars suppress sterol regulatory element binding protein-1c promoter through inhibition of lxr signaling. Mol Endocrinol 2003, 17, 1240-1254.

36. Wang, F.; Mullican, S.E.; DiSpirito, J.R.; Peed, L.C.; Lazar, M.A. Lipoatrophy and severe metabolic disturbance in mice with fat-specific deletion of ppargamma. Proc Natl Acad Sci U S A 2013, 110, 18656-18661.

37. Garg, A. Clinical review\#: Lipodystrophies: Genetic and acquired body fat disorders. J Clin Endocrinol Metab 2011, 96, 3313-3325.

38. Baranowski, M. Biological role of liver x receptors. J Physiol Pharmacol 2008, 59 Suppl 7, 31-55.

39. Cha, J.Y.; Repa, J.J. The liver x receptor (lxr) and hepatic lipogenesis. The carbohydrate-response element-binding protein is a target gene of lxr. J Biol Chem 2007, 282, 743-751.

40. Pettersson, A.M.; Stenson, B.M.; Lorente-Cebrian, S.; Andersson, D.P.; Mejhert, N.; Kratzel, J.; Astrom, G.; Dahlman, I.; Chibalin, A.V.; Arner, P., et al. Lxr is a negative regulator of glucose uptake in human adipocytes. Diabetologia 2013, 56, 2044-2054.

41. Laffitte, B.A.; Chao, L.C.; Li, J.; Walczak, R.; Hummasti, S.; Joseph, S.B.; Castrillo, A.; Wilpitz, D.C.; Mangelsdorf, D.J.; Collins, J.L., et al. Activation of liver $\mathrm{x}$ receptor improves glucose tolerance through coordinate regulation of glucose metabolism in liver and adipose tissue. Proc Natl Acad Sci U S A 2003, 100, 5419-5424.

42. Stenson, B.M.; Ryden, M.; Steffensen, K.R.; Wahlen, K.; Pettersson, A.T.; Jocken, J.W.; Arner, P.; Laurencikiene, J. Activation of liver $\mathrm{x}$ receptor regulates substrate oxidation in white adipocytes. Endocrinology 2009, 150, 4104-4113.

43. Liu, Y.; Yan, C.; Wang, Y.; Nakagawa, Y.; Nerio, N.; Anghel, A.; Lutfy, K.; Friedman, T.C. Liver x receptor agonist t0901317 inhibition of glucocorticoid receptor expression in hepatocytes may contribute to the amelioration of diabetic syndrome in $\mathrm{db} / \mathrm{db}$ mice. Endocrinology 2006, 147, 5061-5068.

44. Commerford, S.R.; Vargas, L.; Dorfman, S.E.; Mitro, N.; Rocheford, E.C.; Mak, P.A.; Li, X.; Kennedy, P.; Mullarkey, T.L.; Saez, E. Dissection of the insulin-sensitizing effect of liver x receptor ligands. Mol Endocrinol 2007, 21, 3002-3012.

(C) 2016 by the authors; licensee Preprints, Basel, Switzerland. This article is an open access article distributed under the terms and conditions of the Creative Commons by Attribution (CC-BY) license (http://creativecommons.org/licenses/by/4.0/). 